\title{
Composite stacking sequence optimization for aeroelastically tailored forward-swept wings
}

\author{
Christopher Bach - Reda Jebari - Andrea Viti - Rob Hewson
}

Received: — / Accepted: -

\begin{abstract}
A method for stacking sequence optimization and aeroelastic tailoring of forward-swept composite wings is presented. It exploits bend-twist coupling to mitigate aeroelastic divergence. The method proposed here is intended for estimating potential weight savings during the preliminary aircraft design stages. A structural beam model of the composite wingbox is derived from anisotropic shell theory and the governing aeroelastic equations are presented for a spanwise discretized forward swept wing. Optimization of the system to reduce wing mass is undertaken for sweep angles of $-35^{\circ}$ to $0^{\circ}$ and Mach numbers from 0.7 to 0.9 . A subset of lamination parameters (LPs) and the number of laminate plies in each pre-defined direction (restricted to $\left.\left\{0^{\circ}, \pm 45^{\circ}, 90^{\circ}\right\}\right)$ serve as design variables. A bi-level hybrid optimization approach is employed, making use of a genetic algorithm (GA) and a subsequent gradientbased optimizer. Constraints are implemented to match lift requirements and prevent aeroelastic divergence, excessive deformations, airfoil stalling and structural failure. A permutation GA is then used to match specific composite ply stacking sequences to the optimum design variables with a limited number of manufacturing constraints considered for demonstration purposes. The optimization results in positive bend-twist coupling and
\end{abstract}

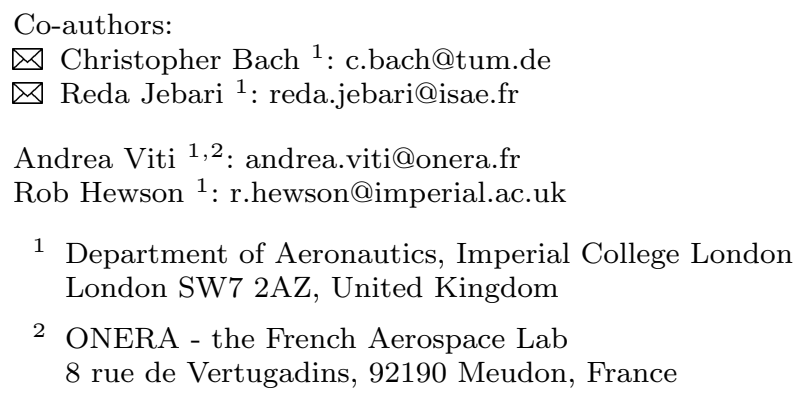

2 ONERA - the French Aerospace Lab 8 rue de Vertugadins, 92190 Meudon, France

a reduced structural mass. Results are compared to an uncoupled reference wing with quasi-isotropic layups and with panel thickness alone the design variables. For a typical geometry and a forward sweep of $-25^{\circ}$ at Mach 0.7 , a wingbox mass reduction of $13 \%$ was achieved.

Keywords Aeroelastic Tailoring · Divergence . Optimization · Composite · Forward-Swept Wing · Lamination Parameters

\section{Introduction}

The use of composite materials in the aerospace industry has been steadily increasing over the past decades. Recent examples of its application are the Airbus A350 and the Boeing 787, with composites accounting for $53 \%$ and $50 \%$ of the structural weight, respectively 18 , 19. Composites benefit from high stiffness and strength along with a low density. A decrease in aircraft structural weight is strongly associated with lower emissions as well as reduced direct operating costs 39. Composites therefore have the potential to play an important role in achieving the goals set by the Advisory Council for Aeronautics Research in Europe, which include a reduction of net $\mathrm{CO}_{2}$ emissions by $50 \%$ by the year 2050, compared to 2005 levels [1].

It is possible to tailor composites for specific applications due to their anisotropic characteristics. They can exhibit couplings between torsion, bending and extension. Aeroelastic tailoring exploits this to reduce weight and improve stability, maneuverability and/or aerodynamic performance. This is particularly beneficial for forward-swept wings (FSW), which have a number of advantageous characteristics. These include a decrease in vortex drag due to weaker vortices at the wing tips 25. Assuming rigid untwisted wings, the onset of stall 
begins at the root and ailerons remain functional at high angles of attack. FSW thus remain maneuverable at high angles of attack [4]. The cabin layout can be improved for smaller aircraft due to the more aft location of the wing root and therefore continuous spar, when compared to a rearward swept wing.

Sweep of wings to mitigate compressibility effects has conventionally been backwards rather than forwards to avoid aeroelastic problems. These include divergence, control reversal, flutter or uncontrollable tip stalling. Overcoming these effects requires an increase in structural stiffness with the associated increase in weight or, as is the focus of this work, by using tailored composites. Composite tailoring can also increase the efficiency and lifetime of wind turbine and helicopter blades. It has been demonstrated that the critical speed could be increased by $12 \%$ 35. Coupling effects between extension and twist can also be applied in the manufacture of helicopter blades as well as in a host of other areas such as in golf clubs or tennis rackets [28].

Preliminary stages of aircraft design rely heavily on empirical relationships and analytical formulations. This work describes a means of estimating potential weight savings achievable by optimizing the stacking sequences of composite wing panels for aeroelastic tailoring. A strip theory aerodynamic model is coupled with a structural model of a thin-walled rectangular composite wingbox, derived from anisotropic shell theory.

The work focuses on mitigating static aeroelastic divergence, which is known to be the dominant aeroelastic problem for FSW and typically occurs before flutter or control reversal [7,36]. Additional aeroelastic problems such as flutter instability or control effectiveness should nevertheless be taken into account in later design stages. More complex and accurate fluid-structure interaction models could then be used for the aeroelastic analyis (reference is made to [14]). For the purpose of initial sizing, however, the use of fast-working models allows the optimization of a large number of design variables to be considered and more fundamentally to explore potential benefits of applying composite tailoring to FSW for preliminary aircraft design. The approach presented in this work can be used to numerically obtain an early estimation of possible weight savings if composite shuffling is undertaken.

\section{Related Work}

Aeroelastic tailoring using composites has been subject to many studies - $[14,22,29,36,46,47,48$, to name a few. Simplifications were often made, such as assuming a span-wise uniform wing or assuming the wing proper- ties vary in a specific way along the span [46].

Previous studies on stacking sequence optimization of laminated composites have introduced lamination parameters (LPs) as design variables, rather than using ply angles and thicknesses. This allows for a reduced design space of continuous design variables, and thus achieves a more efficient optimization than if a large number of discrete design variables are required to define the structure 20. 34 presented a bi-level optimization approach, expressing the laminate stiffness matrices using both a smeared-stiffness model and in terms of LPs. For a given set of ply angles $\left\{0^{\circ}, \pm 45^{\circ}, 90^{\circ}\right\}$, the top-level algorithm optimized the number of plies in each direction along with the associated LPs of each wing panel. In a second step, a stacking sequence was matched to these optima using a permutation GA (PGA). A comprehensive overview of manufacturing constraints and a ply blending algorithm is given by 33 .

Further reference is made to the works of $[21,31,32,9]$ used particle-swarm optimization instead of a PGA in the post-processing step. 14] used a finite element (FE) model to optimize wing stiffnesses with aeroelastic constraints. 4, 6 optimized a flexible wing airfoil using a 2D model and considering flutter and divergence. [15] used bacterial foraging optimization for the aeroelastic tailoring of a composite wing.

The feasible LP region is known to be convex [8, 12, 23], and a closed-form expression has been defined only for a limited number of combinations of the lamination parameters 41. Expressions for a limited set of LPs have been derived by different authors. A method for obtaining an approximate feasible region is presented in 41. [12 developed an approach to define a feasible region for all 12 lamination parameters, which was further extended by [8]. In particular, [13] derived explicit expressions describing the feasible region for ply angles limited to $\left\{0^{\circ}, \pm 45^{\circ}, 90^{\circ}\right\}$. These expressions are used in this work.

\section{Aeroelastic models}

Static aeroelasticity deals with static deformations of the wing and includes the phenomena of divergence and control reversal. The reason why divergence is so critical in forward-swept wings lies in the fact that upwardsbending due to lift increases the wing's local angle of attack, increasing lift further. The phenomenon has been thoroughly explained in [7,22]. Large deformations may occur even before reaching the divergence speed, which is generally undesirable. A local stall and divergence constraint is implemented in the optimization along with an arbitrarily set maximum-twist constraint of 


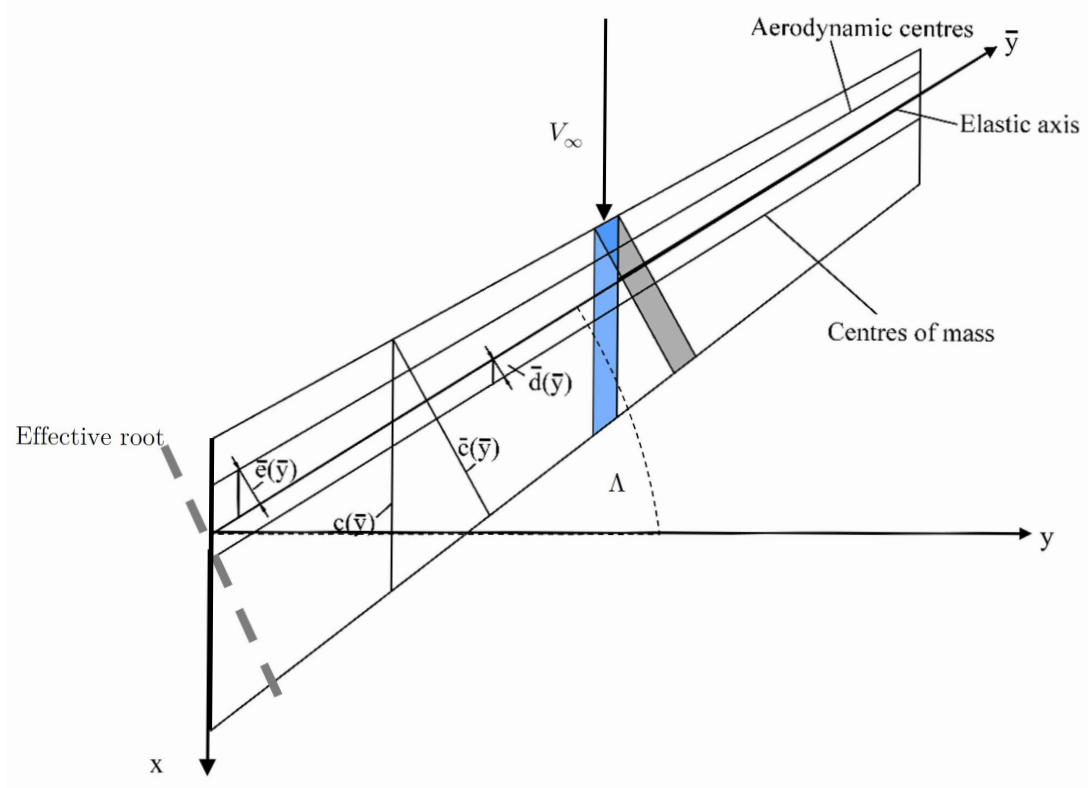

Fig. 1 Geometry of a slender swept-forward wing (notation adapted from [7])

$5^{\circ}$ along the wing. The maximum-twist constraint is used to avoid potentially undesirable large deformations. The influence of drag is neglected as is typical for aeroelastic analysis, where it is usually an order of magnitude smaller than the lift forces.

The wings are modelled as thin-walled cantilever beams (Sect. 5), which is a reasonable approximation for highaspect-ratio wings of passenger jet aircraft 7]. Strip theory is used to describe the wing aerodynamics. It is an approximating model to obtain the lift on an airfoil for inviscid, incompressible flow conditions and an aerodynamically infinite wing span. For more precision, a higher-fidelity model capable of predicting compressibility effects and 3D phenomena such as wing tip vortices that reduce the lift distribution to zero towards the wing tips could be applied [1]. This is of particular interest if aerodynamic considerations beyond that required for the aeroelastic analysis are to be taken into account. However, strip theory allows computationally cheap optimization to be undertaken, especially where the focus of the work is on composite tailoring for the aeroelastic response and preliminary design [15, 5, 29, 30]. The lift is then given by:

$$
\begin{aligned}
& l(y)=q_{\infty} c(y) c_{l}(y) \\
& c_{l}(y)=c_{l 0}(y)+c_{l \alpha}(y) \alpha(y)
\end{aligned}
$$

$c$ denotes the local chord length and $c_{l}$ the local lift coefficient per unit chord. $q_{\infty}$ is the free steam dynamic pressure at cruise conditions, while $\alpha$ denotes the angle of attack. A number of further assumptions are made to develop the aeroelastic models:
- Existence of a straight elastic axis that is swept by the angle $\Lambda$ and serves as the reference wing axis.

- No couplings other than bend-twist, which is achieved by symmetry properties of the cross-section (Sect. 5)

- Small deflections and slender wing

- No rigid pre-twist along the wing (washin/washout)

Furthermore, it is assumed that the wing is clamped to an effective root perpendicular to the elastic axis at the fuselage. This idealization yields sufficiently good results for slender wings [7,46]. Figure 1 gives an overview about the wing geometry and related nomenclature. $d$ denotes the local distance of the centers of mass from the elastic axis, and $e$ is the distance of the aerodynamic centers from the elastic axis. Variables written with a bar (e.g. $\bar{e}(\bar{y})$ ), denote quantities referring to chord-wise segments with respect to the $e(\bar{y})$ frame of reference. Quantities without a bar, e.g. $e(\bar{y})$, refer to the stream-wise direction (this notation is consistent with that used by [7]). The external loads $f_{\text {ext }}, t_{\text {ext }}$ due to lift and gravity are:

$$
\begin{aligned}
& f_{\text {ext }}(y)=q_{\infty} c c_{l}-\mu n g \\
& t_{\text {ext }}(y)=q_{\infty}\left(e c c_{l}+c^{2} c_{m A C}\right)+\mu n g d
\end{aligned}
$$

Variables are transformed from one frame of reference to the other (as described in [7]) by:

$$
\begin{aligned}
& f_{\text {ext }}(\bar{y})=f_{\text {ext }}(y) \cos \Lambda \\
& t_{\text {ext }}(\bar{y})=t_{\text {ext }}(y) \cos \Lambda
\end{aligned}
$$

$n$ is the load factor, which is equal to 1 at cruise. $g$ is the gravitational acceleration, and $\mu$ the mass per unit length. $c_{m A C}$ is the airfoil moment coefficient. The 
following set of differential equations is obtained [7]. Primes $\left({ }^{\prime}\right)$ denote differentiation with respect to $\bar{y}$.

$\bar{M}_{T}^{\prime}(\bar{y})=-t_{e x t}(\bar{y}) \cos \Lambda$

$\bar{M}_{B}^{\prime \prime}(\bar{y})=f_{\text {ext }}(\bar{y})+t_{\text {ext }}^{\prime}(\bar{y}) \sin \Lambda$

The relationship between the torsion and bending moments and the respective curvatures is derived from anisotropic shell theory as described in section 5. It eventually yields the following representation:

$\left[\begin{array}{c}\bar{M}_{T} \\ \bar{M}_{B}\end{array}\right]=\left[\begin{array}{cc}G J & K \\ K & E I\end{array}\right]\left[\begin{array}{c}\bar{\theta}^{\prime} \\ w^{\prime \prime}\end{array}\right]$

$\bar{\theta}$ denotes the elastic twist angle about the elastic axis, and $w$ is the vertical displacement. The coefficients of the cross-sectional stiffness matrix are often denoted as $G J, K$ and $E I$. A non-dimensional coupling constant is used, which is defined as $\kappa=K / \sqrt{E I G J}$. This leads to the following differential equations describing twist and vertical displacement of the wing:

$$
\begin{array}{r}
\left(G J \bar{\theta}^{\prime}\right)^{\prime}+\left(K w^{\prime \prime}\right)^{\prime}=-\left(q_{\infty}\left(e c c_{l}+c^{2} c_{m A C}\right)+\mu n g d\right) \\
\cos ^{2} \Lambda
\end{array}
$$

$\left(E I w^{\prime \prime}\right)^{\prime \prime}+\left(K \bar{\theta}^{\prime}\right)^{\prime \prime}=\left(q_{\infty} c c_{l}-\mu n g\right) \cos \Lambda$

$+\sin \Lambda \cos \Lambda\left(q_{\infty}\left(e c c_{l}+c^{2} c_{m A C}\right)+\mu n g d\right)^{\prime}$

A closed-form solution to equations (7-8) or (10-11) can only be obtained for specific wing properties 7 , 22. Therefore, the Ritz method based on Hamilton's principle has been used. Considering only the homogeneous form of the differential equations is sufficient to obtain a divergence condition [22].

$\int_{t_{1}}^{t_{2}} \delta T-\delta U+\delta W \mathrm{~d} t=0$

In the absence of time dependencies, $\delta T=0$ and $\delta U=$ $\delta W$. Using strip theory, the expressions for the first variation of the potential energy and the virtual work are given by:

$\delta U=\int_{0}^{l} E I w^{\prime \prime} \delta w^{\prime \prime}+K\left(\bar{\theta}^{\prime} \delta w^{\prime \prime}+w^{\prime \prime} \delta \bar{\theta}^{\prime}\right)+G J \bar{\theta}^{\prime} \delta \bar{\theta}^{\prime} \mathrm{d} \bar{y}$

$$
\begin{array}{r}
\delta W=\int_{0}^{l} \delta \bar{\theta}\left(q_{\infty}\left(e c c_{l \alpha} \cos ^{2} \Lambda\right)\left(\bar{\theta} \cos \Lambda-w^{\prime} \sin \Lambda\right)\right) \\
+\delta w\left(q_{\infty} c c_{l \alpha}\left(\bar{\theta} \cos \Lambda-w^{\prime} \sin \Lambda\right)\right) \cos \Lambda \\
\left.+\frac{\mathrm{d}}{\mathrm{d} \bar{y}}\left(q_{\infty} e c c_{l \alpha} \sin \Lambda \cos \Lambda\left(\bar{\theta} \cos \Lambda-w^{\prime} \sin \Lambda\right)\right)\right) \mathrm{d} \bar{y}
\end{array}
$$

The displacements $w$ and $\bar{\theta}$ are expressed as a series of shape functions (Ritz ansatz):

$$
\begin{aligned}
& w(\bar{y})=\sum_{i=1}^{N_{w}} \eta_{i} \psi_{i}(\bar{y}) \\
& \bar{\theta}(\bar{y})=\sum_{j=1}^{N_{\theta}} \xi_{j} \varphi_{j}(\bar{y})
\end{aligned}
$$

The functions $\psi_{i}(\bar{y})$ and $\varphi_{j}(\bar{y})$ must satisfy all essential boundary conditions and be twice continuously differentiable. Completeness and linear independence of the series are also required. The functions are chosen as the free-vibration modes of a clamped-free beam, as presented in 22], pp. 82ff.; 170ff, and satisfy the requirements above.

$\psi_{i}(\bar{y})=\cosh \left(\alpha_{i} \bar{y}\right)-\cos \left(\alpha_{i} \bar{y}\right)-\beta_{i}\left(\sinh \left(\alpha_{i} \bar{y}\right)-\sin \left(\alpha_{i} \bar{y}\right)\right)$

$\varphi_{j}(\bar{y})=\sqrt{2} \sin \left(\frac{\pi(j-1 / 2)}{l}\right)$

The coefficients $\alpha_{i}, \beta_{i}$ are given by table 1 for up to $i=5$, where $l=b /(2 \cos \Lambda)$ is the length of the wing and $b$ is the wingspan.

Table 1 Values of $\alpha_{i}, \beta_{i}$. 22, p.83

\begin{tabular}{lll}
\hline \hline $\mathrm{i}$ & $\alpha_{i} l$ & $\beta_{i}$ \\
\hline 1 & 1.87510 & 0.734096 \\
\hline 2 & 4.69409 & 1.01847 \\
\hline 3 & 7.85476 & 0.999224 \\
\hline 4 & 10.9955 & 1.00003 \\
\hline 5 & 14.1372 & 0.999999 \\
\hline
\end{tabular}

By inserting equations (15-18) into (12), a system of equations can be obtained. Let $\boldsymbol{\chi}$ be the vector of generalised displacements:

$\underline{\chi}=\left[\eta_{1}, \ldots, \eta_{N_{w}}, \xi_{1}, \ldots, \xi_{N_{\theta}}\right]^{T}$

The obtained matrix system is of the form:

$$
[\boldsymbol{A}] \underline{\boldsymbol{\chi}}=\left(\left[\boldsymbol{A}_{\text {stiff }}\right]+q_{\infty}\left[\boldsymbol{A}_{\text {aero }}\right]\right) \underline{\boldsymbol{\chi}}=0
$$

This allows the divergence dynamic pressure $q_{D}$ as a solution of an eigenvalue problem to be obtained:

$$
\frac{1}{q_{\infty}} \underline{\chi}=[\mathcal{M}] \underline{\chi}
$$

The largest positive eigenvalue of the matrix $[\mathcal{M}]$ is an approximate representation of $1 / q_{D} 22$. The calculation of $[\mathcal{M}]$, which combines aerodynamic and stiffness terms, is given in appendix A A presentation of 
the underlying theory is given by [27], where a closedform expression of flutter- and divergence-type instability speeds is derived.

The aeroelastic equations are solved numerically to obtain $\bar{\theta}$ and $w^{\prime}$ as well as internal forces and moments along the wingspan. $c_{l}$ could be evaluated by other means than strip theory as is used in this case. Here, the inhomogeneous equations need to be considered. After non-dimensionalizing with $\eta:=\bar{y} / l$ and exploiting the relationship $Q(\bar{y})=\bar{M}_{B}^{\prime}(\bar{y})$, equation (8) can be transformed to give:

$$
\begin{array}{r}
Q(\eta)^{\prime}=l\left(q_{\infty} c(\eta) c_{l}\left(\eta, \bar{\theta}(\eta), w^{\prime}(\eta)\right)-\mu(\eta) n g\right) \cos \Lambda \\
+\frac{\mathrm{d}}{\mathrm{d} \eta}\left(q_{\infty} e(\eta) c(\eta) c_{l}\left(\eta, \bar{\theta}(\eta), w^{\prime}(\eta)\right)\right. \\
\left.+q_{\infty} c(\eta)^{2} c_{m A C}(\eta)+\mu(\eta) n g d(\eta)\right) \sin \Lambda \cos \Lambda
\end{array}
$$

Note that the variables $c, c_{l}, e, d, \mu, E I, G J, K$ and $c_{m A C}$ in (24) are all functions of $\eta$. In particular, $c_{l}$ depends on $\eta, \bar{\theta}(\eta)$ and $w^{\prime}(\eta)$.

The variables $\left(\gamma_{1}, \gamma_{2}, \gamma_{3}, \gamma_{4}, \gamma_{5}\right)$ are chosen as follows:

$$
\left[\begin{array}{c}
\gamma_{1} \\
\gamma_{2} \\
\gamma_{3} \\
\gamma_{4} \\
\gamma_{5}
\end{array}\right]=\left[\begin{array}{c}
Q-\sin \Lambda \cos \Lambda\left(q_{\infty} e c c_{l}+q_{\infty} c^{2} c_{m A C}+\mu n g d\right) \\
\bar{M}_{B}(\eta) \\
\bar{M}_{T}(\eta) \\
w^{\prime}(\eta) \\
\bar{\theta}(\eta)
\end{array}\right]
$$

The resulting boundary value problem (BVP) can then be written as:

$\underline{\gamma}^{\prime}(\eta)=\left[\begin{array}{c}l\left(q_{\infty} c c_{l}-\mu n g\right) \cos \Lambda \\ l \gamma_{1}+l \sin \Lambda \cos \Lambda\left(q_{\infty} e c c_{l}+q_{\infty} c^{2} c_{m A C}+\mu n g d\right) \\ -l \cos ^{2} \Lambda\left(q_{\infty}\left(e c c_{l}+c^{2} c_{m A C}\right)+\mu n g d\right) \\ \frac{l^{2}\left(G J \gamma_{2}-K \gamma_{3}\right)}{E I G J-K^{2}} \\ \frac{l\left(E I \gamma_{3}-K \gamma_{2}\right)}{E I G J-K^{2}}\end{array}\right]$

The external boundary conditions of the clamped-free beam are given by:

$$
\begin{aligned}
& \gamma_{4}(0)=\gamma_{5}(0)=0 \\
& \gamma_{2}(1)=\gamma_{3}(1)=0 \\
& \left.\left(\gamma_{1}+\sin \Lambda \cos \Lambda\left(q_{\infty}\left(e c c_{l}+c^{2} c_{m A C}\right)+\mu n g d\right)\right)\right|_{\eta=1}=0
\end{aligned}
$$

This BVP is solved numerically, in this case using a boundary value problem solver implemented in MATLAB ( $b v p 4 c$ function) [38. The internal forces and moments are then obtained from (23). The aeroelastic models were compared to the closed-form solution for a uniform, coupled forward-swept wing, and the analytical approximation of the divergence boundary [22], pp.
161-167. The models showed good agreement. Forwardsweep and negative coupling constants $\kappa, K$ were found to significantly reduce the divergence dynamic pressure. As expected, positive coupling constants were found to increase $q_{D}$ and could be exploited for aeroelastic tailoring.

\section{Lamination parameters}

The laminate stiffness matrices $[\boldsymbol{A}],[\boldsymbol{B}]$ and $[\boldsymbol{D}]$ are used to relate the resultant forces and moments to the mid-plane strains and curvatures $[26] .[\boldsymbol{A}],[\boldsymbol{B}]$ and $[\boldsymbol{D}]$ are the extensional, coupling and bending stiffness matrices, respectively. They can be expressed using 12 lamination parameters and invariant laminate stiffnesses 17,43. Assuming all plies are of the same material, the LPs are defined as normalized through-thickness integrals of ply angles $\theta$ [45]:

$$
\begin{aligned}
& {\left[\begin{array}{l}
V_{1}^{A} \\
V_{2}^{A} \\
V_{3}^{A} \\
V_{4}^{A}
\end{array}\right]=\frac{1}{h} \int_{-h / 2}^{h / 2}\left[\begin{array}{l}
\cos 2 \theta \\
\sin 2 \theta \\
\cos 4 \theta \\
\sin 4 \theta
\end{array}\right] \mathrm{d} z} \\
& {\left[\begin{array}{l}
V_{1}^{B} \\
V_{2}^{B} \\
V_{3}^{B} \\
V_{4}^{B}
\end{array}\right]=\frac{4}{h^{2}} \int_{-h / 2}^{h / 2}\left[\begin{array}{l}
\cos 2 \theta \\
\sin 2 \theta \\
\cos 4 \theta \\
\sin 4 \theta
\end{array}\right] z \mathrm{~d} z} \\
& {\left[\begin{array}{l}
V_{1}^{D} \\
V_{2}^{D} \\
V_{3}^{D} \\
V_{4}^{D}
\end{array}\right]=\frac{12}{h^{3}} \int_{-h / 2}^{h / 2}\left[\begin{array}{l}
\cos 2 \theta \\
\sin 2 \theta \\
\cos 4 \theta \\
\sin 4 \theta
\end{array}\right] z^{2} \mathrm{~d} z}
\end{aligned}
$$

where $h$ is the laminate thickness. The stiffness matrices may then be expressed as following [23].

$$
\begin{aligned}
& {[\boldsymbol{A}]=h\left(\left[\boldsymbol{\Gamma}_{0}\right]+\left[\boldsymbol{\Gamma}_{1}\right] V_{1}^{A}+\left[\boldsymbol{\Gamma}_{2}\right] V_{2}^{A}+\left[\boldsymbol{\Gamma}_{3}\right] V_{3}^{A}+\left[\boldsymbol{\Gamma}_{4}\right] V_{4}^{A}\right)} \\
& {[\boldsymbol{B}]=\frac{h^{2}}{4}\left(\left[\boldsymbol{\Gamma}_{1}\right] V_{1}^{B}+\left[\boldsymbol{\Gamma}_{2}\right] V_{2}^{B}+\left[\boldsymbol{\Gamma}_{3}\right] V_{3}^{B}+\left[\boldsymbol{\Gamma}_{4}\right] V_{4}^{B}\right)} \\
& {[\boldsymbol{D}]=\frac{h^{3}}{12}\left(\left[\boldsymbol{\Gamma}_{0}\right]+\left[\boldsymbol{\Gamma}_{1}\right] V_{1}^{D}+\left[\boldsymbol{\Gamma}_{2}\right] V_{2}^{D}+\left[\boldsymbol{\Gamma}_{3}\right] V_{3}^{D}+\left[\boldsymbol{\Gamma}_{4}\right] V_{4}^{D}\right)}
\end{aligned}
$$

The material invariant matrices $\left[\boldsymbol{\Gamma}_{i}\right]$ and the material invariants $U_{i}$ are defined in appendix $\mathrm{B}$. It is worth noting that for the case of ply angles limited to $\left\{0^{\circ}, \pm 45^{\circ}\right.$, $\left.90^{\circ}\right\}$, the $\sin 4 \theta$ terms will always be equal to zero, resulting in $V_{4}^{(A, B, D)}=0$. All $V_{i}^{B}$ terms are equal to zero, as only symmetric laminates are considered. This is done to avoid thermal distortions after cooling down from the curing temperature during the manufacturing process. 


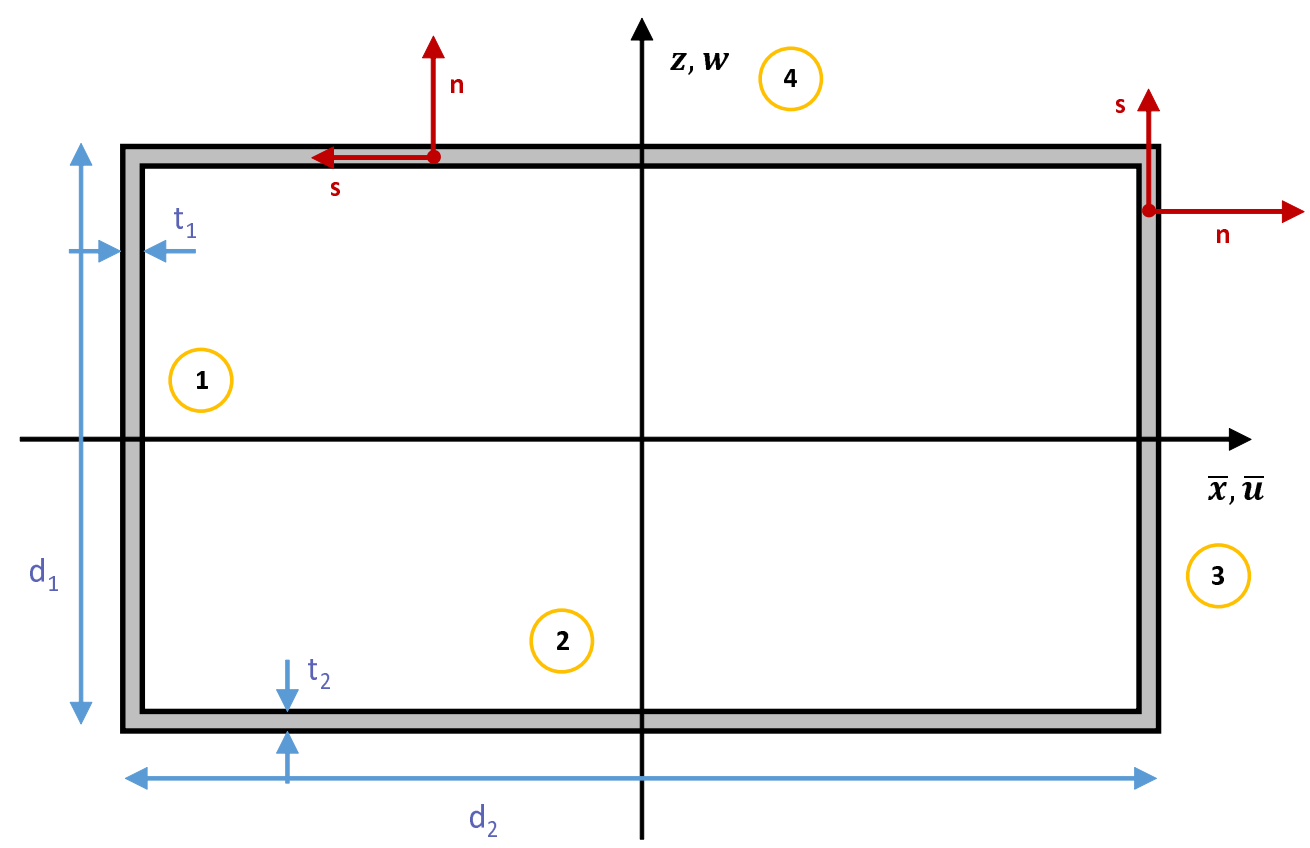

Fig. 2 Cross-section description

\section{Structural model}

The wing is modelled as a thin-walled cantilever beam with rectangular cross-sections. The wingbox is assumed to carry all loads, while the leading and trailing edges are only taken into account for their mass and their aerodynamic properties. 3] presented an approach to analyze the mechanical behavior of thin-walled composite beams. Torsion has been taken into account in the model [37. 40, used these results to derive a relation between the beam's loads and deformation. A simplified method of their work for rectangular cross-sections and without non-classical effects has been used here.

Figure 2 represents a cross-section of each partition of the thin-walled box beam. The stacking sequence of each panel is obtained by counting the plies from the inside to the outside of the cross-section, with the $0^{\circ}$ direction being aligned with the elastic axis. The thicknesses of the walls, $t_{1}$ and $t_{2}$, are assumed to be small compared to the beam's other dimensions. $d_{1}$ is the height and $d_{2}$ the width of the beam. The model does not take into account the effects of restrained warping and transverse shear. 30 found this leads to either a conservative estimate of the resulting divergence speed, or the difference was less than $2 \%$ for slender structures such as the wingbox considered here.

Further assumptions are made in accordance to [16] and 3, which are specific to composite thin-walled beams. There is no deformation in the cross-section's plane, in other words the in-plane warping is neglected. Each wall of the box is considered to be a thin plate and its behavior governed by the linear elastic rules of classical laminate theory. The behavior of the box beam thus arises from the combined behavior of its thin-plate walls. Normal stress $N_{s}$ and bending moment $M_{s}$ about $\underline{s}$ are neglected in this bending problem. A $9 \times 9$ global stiffness matrix is obtained, relating the beam's loads to its deformations. This reduces to a $4 \times 4$ matrix by neglecting non-classical effects 24]. The final shape of the global stiffness matrix $\left[\boldsymbol{K}_{r}\right]$ is:

$$
\left[\begin{array}{c}
\bar{N}(\bar{y}) \\
\bar{M}_{T}(\bar{y}) \\
\bar{M}_{B, \bar{x}}(\bar{y}) \\
M_{B, z}(\bar{y})
\end{array}\right]=\underbrace{\left[\begin{array}{llll}
K_{1,1} & K_{1,2} & K_{1,3} & K_{1,4} \\
K_{2,1} & K_{2,2} & K_{2,3} & K_{2,4} \\
K_{3,1} & K_{3,2} & K_{3,3} & K_{3,4} \\
K_{4,1} & K_{4,2} & K_{4,3} & K_{4,4}
\end{array}\right]}_{\left[\boldsymbol{K}_{r}\right]}\left[\begin{array}{c}
\bar{v}^{\prime}(\bar{y}) \\
\bar{\theta}^{\prime}(\bar{y}) \\
w^{\prime \prime}(\bar{y}) \\
\bar{u}^{\prime \prime}(\bar{y})
\end{array}\right]
$$

with $\bar{v}$ as the extensional displacement in $\bar{y}$-direction and $\bar{\theta}$ and $w$ as the twist angle and the vertical displacement in $z$-direction, respectively (see section 3 ). $\bar{u}$ denotes the horizontal displacement in $\bar{x}$-direction.

Making the stacking sequences of the top and bottom panels antisymmetric about one another produces the greatest bend-twist coupling [2], and has been derived from the expressions of the stiffness matrix obtained by the current model. The same feature applied to the side panels would produce a coupling between the bending around the y-axis and the twist, which is undesirable. Hence, the side panels were made symmetric one to another. Balanced panels would cancel the coupling, so 
they were left unbalanced. By taking the symmetries into account, the stiffness matrix simplifies to [24]:

$\left[\boldsymbol{K}_{r}\right]=\left[\begin{array}{cccc}E A & 0 & 0 & 0 \\ 0 & G J & K & 0 \\ 0 & K & E I_{f} & 0 \\ 0 & 0 & 0 & E I_{l}\end{array}\right]$

This corresponds to equation (9) in section 3 . The stiffness terms are:

$$
\begin{aligned}
& E A=2 d_{1} A_{z}^{(1)}+2 d_{2} A_{z}^{(2)} \\
& G J=2\left(\frac{d_{1} d_{2} t_{1} t_{2}}{d_{1} t_{2}+d_{2} t_{1}}\right)^{2}\left(\frac{d_{1}}{t_{1}^{2}} A_{s}^{(1)}+\frac{d_{2}}{t_{2}^{2}} A_{s}^{(2)}\right) \\
& +8\left(d_{1} D_{s}^{(1)}+d_{2} D_{s}^{(2)}\right)
\end{aligned}
$$

Where the superscripts refer to the panel number, and the reduced stiffness matrix terms $A_{x}^{(i)}$ and $D_{x}^{(i)}$ are function of the $[\boldsymbol{A}]$ and the $[\boldsymbol{D}]$ stiffness matrices of each panel. Their exact expression is available in appendix C, $E A$ is the extensional stiffness, $G J$ and $K$ represent the torsional and the coupling stiffness, respectively. $E I_{f}$ and $E I_{l}$ are the two bending stiffnesses about the different axes.

Comparison has been made between the model derived here and that of [2, which was verified using FE simulation and test data, but neglects the effects of the $[\boldsymbol{D}]$ matrix. For the type of composite beams considered in this work, good agreement of the stiffness matrix terms was found. The difference in $E A, G J, E I_{f}$ and $E I_{l}$ was less than $1 \%$ between the models, while the difference between the bend-twist couplings $K$ was less than $8.5 \%$ and may be due to 2$]$ neglecting the role of the $[\boldsymbol{D}]$ matrix.

\section{Optimization problem}

The wings are discretized into five equidistant segments with piecewise constant ply layups and stiffness properties. This yields a total of 20 composite wing panels. The stacking sequences can vary independently from panel to panel. The optimization process comprises a bi-level approach, which uses the number of plies in the pre-defined directions $\left\{0^{\circ}, \pm 45^{\circ}, 90^{\circ}\right\}$ and $V^{D}$ lamination parameters as design variables. A genetic algorithm
Table 2 Chosen parameters (aircraft configuration)

\begin{tabular}{ll|ll}
\hline \hline Wingspan & $30 \mathrm{~m}$ & Material & T300-914 \\
Root chord & $5 \mathrm{~m}$ & Safety factor & 1.5 \\
Taper ratio & 0.25 & Required lift & $600,000 \mathrm{~N}$ \\
Airfoil & 65410 & Wingbox position & $1 \mathrm{~m}$ \\
& & (LE to wingbox) & \\
\hline
\end{tabular}

Table 3 Dynamic pressures at $\mathrm{h}=10,000 \mathrm{~m}$

\begin{tabular}{llllll}
\hline \hline$M_{\infty}$ & 0.70 & 0.75 & 0.80 & 0.85 & 0.90 \\
$V_{\infty}[\mathrm{m} / \mathrm{s}]$ & 209.6 & 224.6 & 239.5 & 254.5 & 269.5 \\
$q_{\infty}[\mathrm{Pa}]$ & 9,060 & 10,400 & 11,833 & 13,358 & 14,976 \\
\hline
\end{tabular}

was used, followed by gradient-based optimizer (SQP) to verify the optima. A permutation GA is then used to obtain a stacking sequence from the number of plies and $V^{D}$ LPs.

Optimization was undertaken for a range of sweep angles, $\Lambda$, from $-35^{\circ}$ to $0^{\circ}$, cruise Mach numbers $M_{\infty}$ between 0.70 and 0.90 , and parameters described in table 2. The parameters represent a fictional passenger aircraft with linearly tapered wings, approximately the size of an Airbus A320. T300-914 composite material properties are taken from [10,24,42. The ambient dynamic pressure $q_{\infty}$ corresponding to the the specified Mach numbers is calculated from the International Civil Aviation Organization Standard Atmosphere (ISA), for a cruise altitude of $10,000 \mathrm{~m}$ [44. At this altitude, the temperature is obtained as $T=223 \mathrm{~K}$, resulting in an air density of $\rho=0.41 \mathrm{~kg} / \mathrm{m}^{3}$. Air speeds and dynamic pressures for different Mach numbers are given in table 3 . The optimization process was implemented in MATLAB, using the functions $g a$ and fmincon.

The hybrid optimization process starts by creating an initial population for the LP-based GA, with $\geq 10 \%$ plies in each direction. The initial population also exhibits a decreasing panel thickness from root to tip and respects the feasible LP region. For each generation, the genetic algorithm then evaluates the fitnesses defined as the wingbox mass. Violations of constraints are implemented as penalties applied to the objective function.

The objective is to minimize the structural mass of the composite wingbox. It is obtained from the resulting panel thicknesses and the cross-sectional dimensions:

$m=\int_{0}^{l} \mu_{\text {wingbox }}(\bar{y}) \mathrm{d} \bar{y} \approx 2 \sum_{i=1}^{N} \rho \frac{l}{N}\left(d_{1} t_{1}+d_{2} t_{2}\right)_{i}$

where $N=5$ is the number of discretization segments of the wing, $\rho$ is the material density and $\left(d_{1}, d_{2}, t_{1}, t_{2}\right)_{i}$ are the cross-sectional dimensions and skin thicknesses of the wingbox at the i-th segment. 
Table 4 Optimization algorithms in the lamination parameter based optimization model

\begin{tabular}{|c|c|c|c|}
\hline Setup & Genetic Algorithm ( $g a)$ & Gradient based (fmincon) & Permutation GA (modified $g a$ ) \\
\hline Objective & Minimize mass & Minimize mass & Match stacking sequence to $V^{D} \mathrm{~s}$ \\
\hline $\begin{array}{l}\text { Design } \\
\text { variables }\end{array}$ & $\begin{array}{l}\text { Number of plies in each } \\
\text { direction and } V^{D} \text { parameters } \\
\text { for each panel. } \alpha_{r} \text { is part of } \\
\text { internal optimization loop }\end{array}$ & $\begin{array}{l}\text { Number of plies in each } \\
\text { direction and } V^{D} \text { parameters } \\
\text { for each panel; } \alpha_{r}\end{array}$ & $\begin{array}{l}\text { Stacking sequence, represented by } \\
\text { an array of integers. }[1,2,3,4] \text { encodes } \\
\text { the available ply angles }\left[0^{\circ}, 45^{\circ}, 90^{\circ},\right. \\
\left.45^{\circ}\right] \text {. Correct number of plies in each } \\
\text { direction is set naturally, the algo- } \\
\text { rithm only shuffles the sequence. }\end{array}$ \\
\hline Constraints & $\begin{array}{l}\text { Feasible LP region, }>10 \% \\
\text { plies in each direction } \\
\text { No divergence or stalling, less } \\
\text { than } 5^{\circ} \text { wing twist. Produced } \\
\text { lift within } 1 \% \text { of required lift. } \\
\text { No structural failure. }\end{array}$ & $\begin{array}{l}\text { Feasible LP region, }>10 \% \\
\text { plies in each direction } \\
\text { No divergence or stalling, less } \\
\text { than } 5^{\circ} \text { wing twist. Produced } \\
\text { lift within } 1 \% \text { of required lift. } \\
\text { No structural failure. }\end{array}$ & $\begin{array}{l}\text { Naturally satisfies manufacturing } \\
\text { rules implemented in the other algo- } \\
\text { rithms. Further rules can be imple- } \\
\text { mented. }\end{array}$ \\
\hline
\end{tabular}

The optimization is subject to a number of constraints given below:

- No divergence

- No stalling due to flow separation, i.e. $\alpha<\alpha_{\text {stall }}$ at all wing span locations

- Twist angle $\bar{\theta}(\bar{y}) \leq 5^{\circ}$ at all wing span locations

- Lift equal to lift required $\pm 1 \%$

- Ply percentage in each pre-defined direction $\geq 10 \%$

- No structural failure as estimated by the Tsai-Hill criterion (first-ply-failure, FPF)

- Feasible LP region as described by 13

The undeformed wing angle of attack $\alpha_{r}$ was adjusted to ensure that the desired lift at the cruise condition was attained within a tolerance of $1 \%$, this was facilitated by the monotonic increase in lift with undeformed angle of attack. Bounds for $\alpha_{r}$ were set to keep it between $-1^{\circ}$ and $5^{\circ}$. The lift was obtained by integrating the sectional lift over both wings. The wing deformations and the local angle of attack were obtained using the numerical solution of the BVP. The wing stiffnesses and other properties were expressed as functions of $\bar{y}$. This requires the calculation of the $[\boldsymbol{A}],[\boldsymbol{B}],[\boldsymbol{D}]$ matrices of each laminate (Sect. 5). The Ritz method was then used to determine if divergence occurs. The deformations and internal forces and moments along the wing were evaluated and a check made for structural failure made.

A population size of 500 individuals was used, with a stop-condition of 100 stalled generations. The optimum point obtained from the GA was passed to the gradient-based optimization model. The gradient-based algorithm (SQP) then refines this point.

On convergence of the optimum number a stacking sequence was matched to the number of plies and $V^{D}$ terms of each panel. Further manufacturing rules such as ply blending from panel to panel could be incorporated here as demonstrated by 33 . To accomplish the stacking sequence matching, the number of plies has to be rounded to an even integer number to ensure symmetric layups for each panel and avoid structural failure. This is not possible with odd numbers of plies. Hence, the final mass of the wingbox structure after the stacking sequence matching will be higher than for the initial optimum set of design variables obtained from the continuous optimization.

The PGA for matching a stacking sequence to the optimum LPs uses an integer array with values between 1 and 4 encoding the stacking sequences. An initial population was created. Its individuals were random permutations with the correct number of plies in each direction. Crossover operations are not present in a PGA, as they could change the number of plies in each direction and produce undesirable results.

The mutation operation swaps the positions of a certain number of plies within the stack of an individual. Moreover, the algorithm was designed so that it naturally only produces symmetric layups. Additional manufacturing rules such as ply blending could be included. As the number of plies in each direction stays the same and the stacks are symmetric, the $V^{A}$ parameters will always be identical, and the $V^{B}$ will be equal to zero. Only the $V^{D}$ parameters need to be matched. The fitness function is defined as a Euclidean norm:

$f(\underline{\boldsymbol{V}})^{D}=\sqrt{\sum_{i=1}^{4}\left(\Delta V_{i}^{D}\right)^{2}}$
$\Delta \underline{\boldsymbol{V}}^{D}=\underline{\boldsymbol{V}}_{\text {optimum }}^{D}-\underline{\boldsymbol{V}}^{D}$

The optimization was undertaken using parallel processing on 16 cores of the Linux Cluster at the Department of Aeronautics, Imperial College London. 

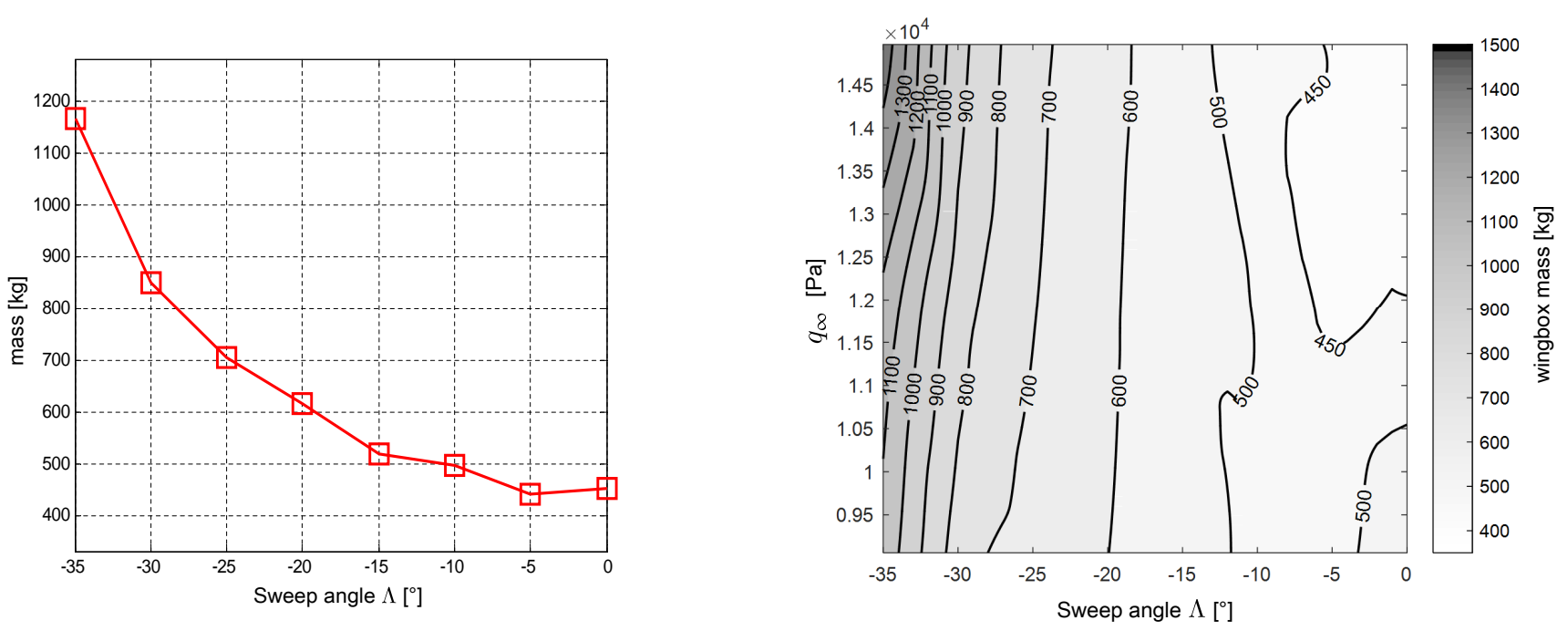

Fig. 3 Obtained plots of optimum wingbox mass vs. sweep angle $\Lambda$ and dynamic pressure $q_{\infty}$. Simulations were run for different $q_{\infty}$ corresponding to Mach numbers $0.7,0.8,0.9$, and for $\Lambda$ between $-35^{\circ}$ and $0^{\circ}$ using $5^{\circ}$-steps. 2D cut (right) for $M_{\infty}=0.8$ and contour plot (left).

\section{Results and discussion}

Results show that the optimum structural mass increases for larger forward-sweep. The wingbox mass increases most rapidly as the forward sweep angle increases beyond $-25^{\circ}$ as shown in (figure 3). A forward-sweep of $-20^{\circ}$ exhibited a mass increase of $6.4 \%$ at Mach $0.7,28 \%$ at Mach 0.8 and $36 \%$ at Mach 0.9 , compared to the unswept case. The gradient-based second stage of the optimization process using the SQP algorithm revealed that the LP-based GA had converged to an optimum in every simulation that was run. Small improvements were achieved when using continuous variables, but the mass was found to be the same after rounding up the number of plies in each panel to even numbers. While the GA was used to achieve a global optimization, the algorithm may still have converged to a local optimum in some cases.

For low amounts of forward-sweep, the optimum structural mass was found to show a small sensitivity to the ambient dynamic pressure $q_{\infty}$, which is related to the flight Mach number $M_{\infty}$ at given flying conditions (without considering compressibility effects). At large forward-sweep angles, there is a trend of increasing the structural mass even further for higher values of $q_{\infty}$. This can be explained by the divergence constraint, which grows more and more dominant for higher amounts of forward-sweep. The wings must neither diverge nor exhibit large deformations up to the specified dynamic pressure. This trend is no longer present for small angles of forward-sweep.

The optimum distributions of $E I, G J$ and $K$ were an- alyzed. The results are plotted against $\bar{y}$ in figure 5 for $\Lambda=-15^{\circ}$ and $M_{\infty}=0.8$. As expected, the stiffnesses decrease towards the tip, as the chord and moments are reducing. Flexural and torsional rigidities are of similar magnitude, while $\kappa$ assumes only positive values. As established earlier, positive $\kappa$ cause pitch-down twisting when bending upwards and thus mitigate aeroelastic divergence. Large values of $\kappa$ occur especially towards the center and tip of the wing, where larger twists and deflections occur.

The stiffnesses were approximated as constant over each discretization segment. Accuracy can be improved using a higher-order interpolation or finer discretization of the wing. A general trend to higher values of $\kappa$ for larger amounts of forward-sweep was observed, especially for the centremost panels.

An interesting phenomenon can be seen in figure 4 The lift distribution is shown along with the varying chord and section lift coefficient along the wing. The lift coefficient is nearly constant for low forward-sweep angles, yielding a lift distribution that decreases almost linearly with the chord (4a). As the amount of forward-sweep increases, the loads shift more and more towards the center of the wing (4a-c). This is caused by a bendinginduced increase of the angle of attack towards the tip (figure 6). The lift distribution does not drop to zero at the wing tip, as the simple strip theory was used to approximate the wing aerodynamics. More accurate aerodynamic models would produce lower loads towards the tip. However, the lower internal moments at the tip lead to lower sensitivity as noted above. 

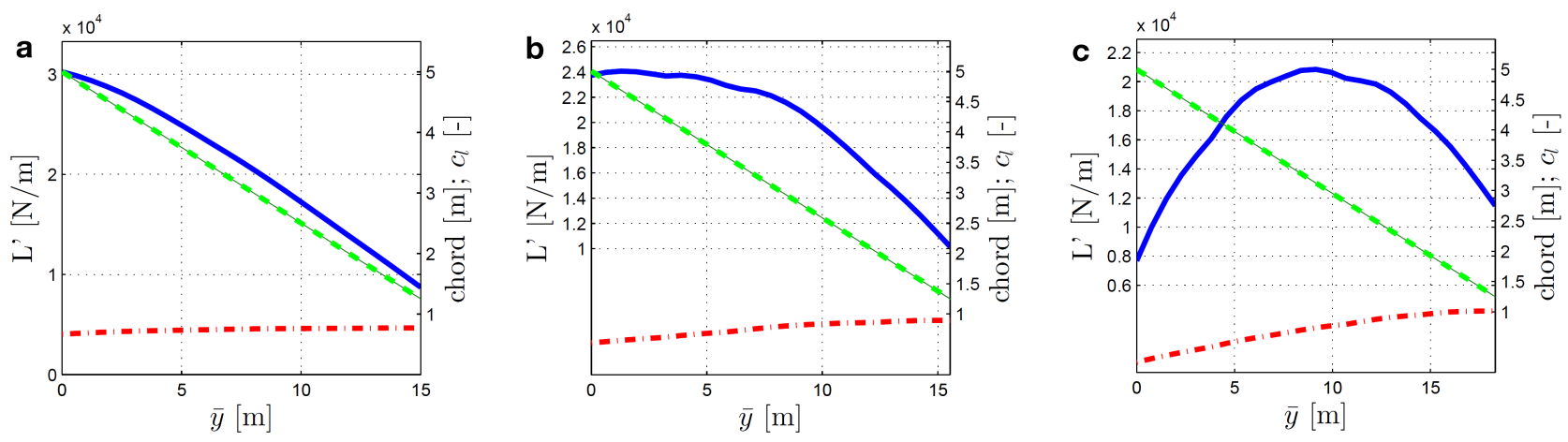

$$
\begin{aligned}
& \text { - }=\text { Section Lift L' } \\
& \text {, - - . Section Lift coefficient c, }
\end{aligned}
$$

Fig. 4 Lift distributions of optimum results, obtained for a cruise $M_{\infty}=0.7$ and $\Lambda=0^{\circ}(\mathbf{a}),-15^{\circ}$ (b) and $-30^{\circ}$ (c).
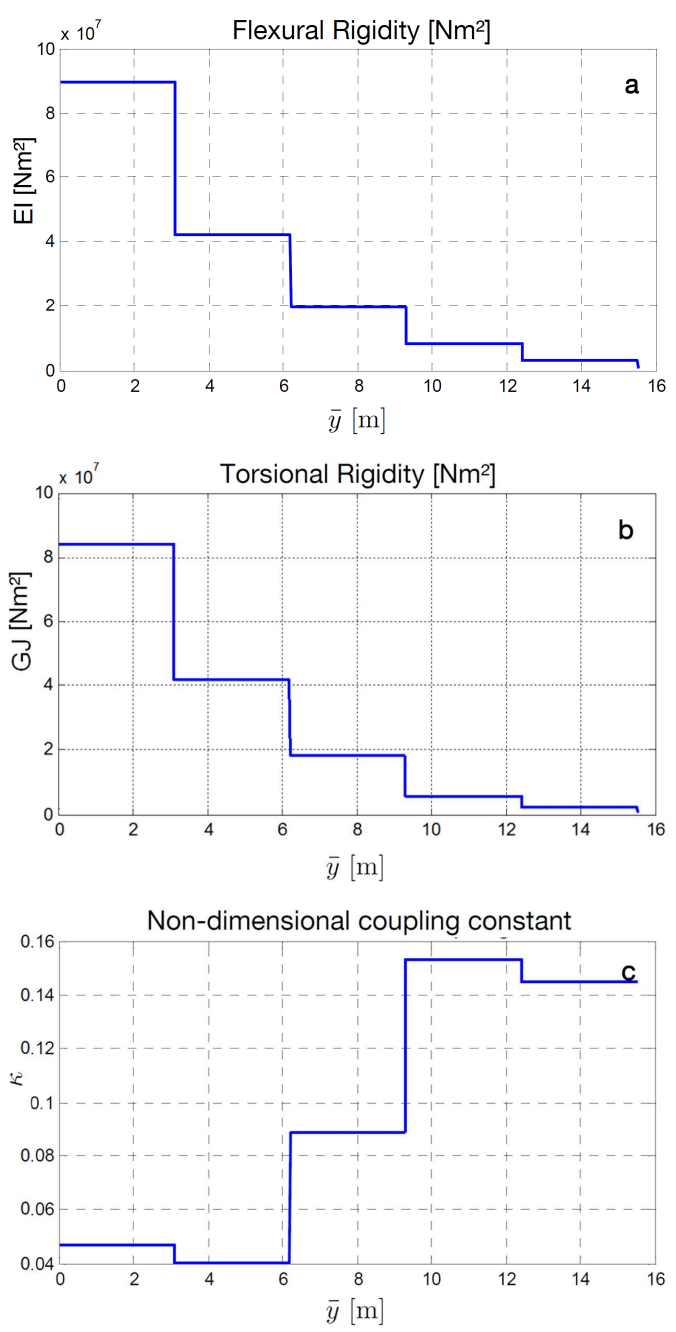

Fig. 5 Optimum stiffnesses $E I, G$ and $\kappa$. Results obtained for $\Lambda=-15^{\circ}, M_{\infty}=0.8$
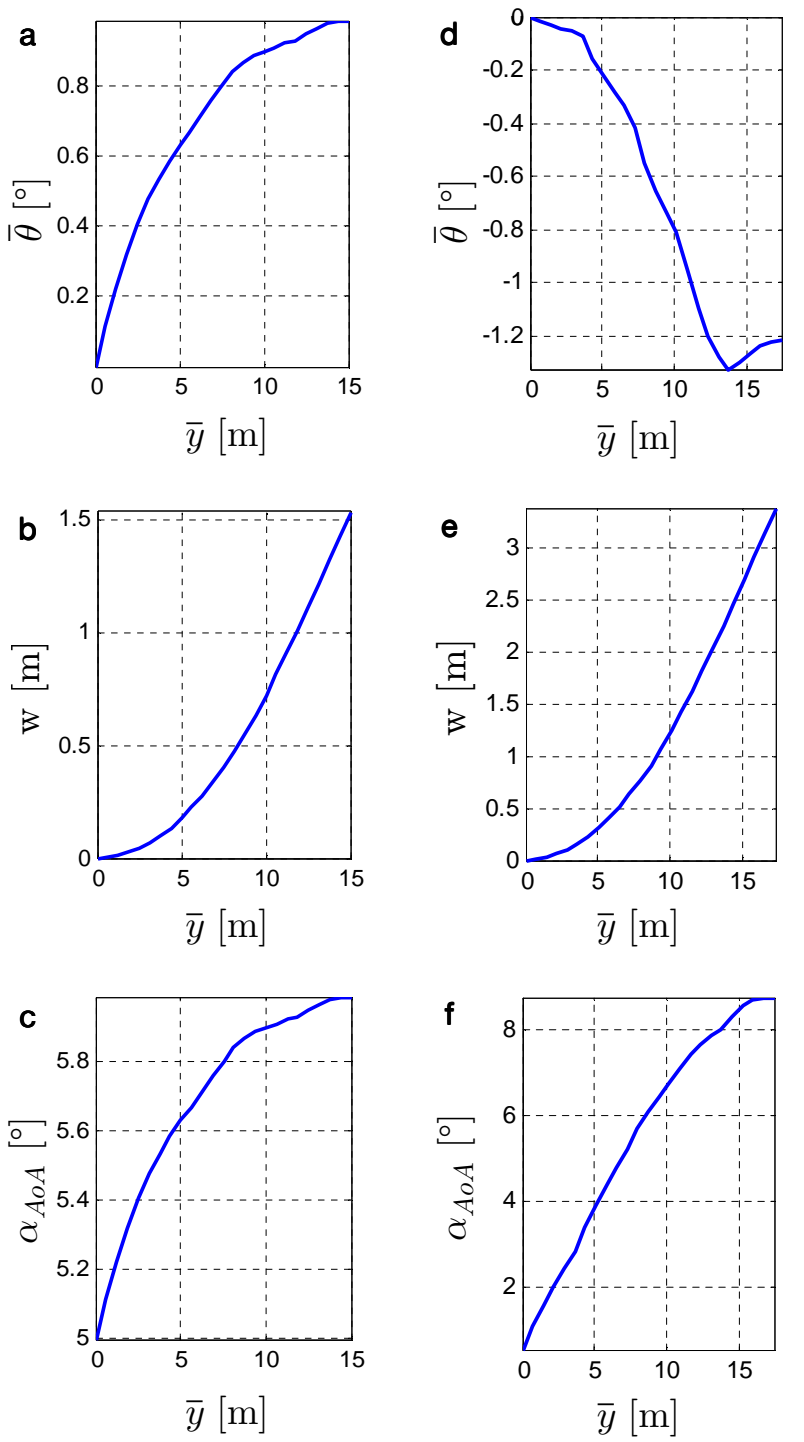

Fig. 6 Displacements and angle of attack along the wing for different sweep angles. a-c: $\Lambda=0^{\circ}$ and $M_{\infty}=0.7$. d-f: $\Lambda=-30^{\circ}$ and $M_{\infty}=0.7$ 
At Mach 0.7, the optimum $\alpha_{r}$ is at the maximum permitted value of $5^{\circ}$ for an unswept wing.(figure 6 a-c). The optimum for a forward-sweep of $-30^{\circ}$ is found to be $\alpha_{r}=0.6^{\circ}$ (figure 6d-f). The lift constraint thus turns out to be fairly dominant for the optimization if the wing area cannot be changed.

Interestingly, the side panels, i.e. front and rear spar, tend to be thicker than the top and bottom panels. This difference in thickness grows more and more prominent towards the wing tip (figure 7). This could be due to the loads acting on the wing in shear and bending, and the poor performance of composites in shear compared to tension and compression. The top and bottom panels mainly experience tension and compression due to bending, while the side panels carry the shear loads.

A noticeable insight is the large fraction of $45^{\circ}$ plies in the top panel. Figure 8 illustrates this behavior for a Mach number of 0.8 , but the same trend can be observed for all simulations. It is responsible for creating positive coupling constants $\mathrm{K}$ and $\kappa$ to mitigate aeroelastic divergence. Due to the asymmetric stiffness design (Sect. 5), the number of $45^{\circ}$ plies in the top panel

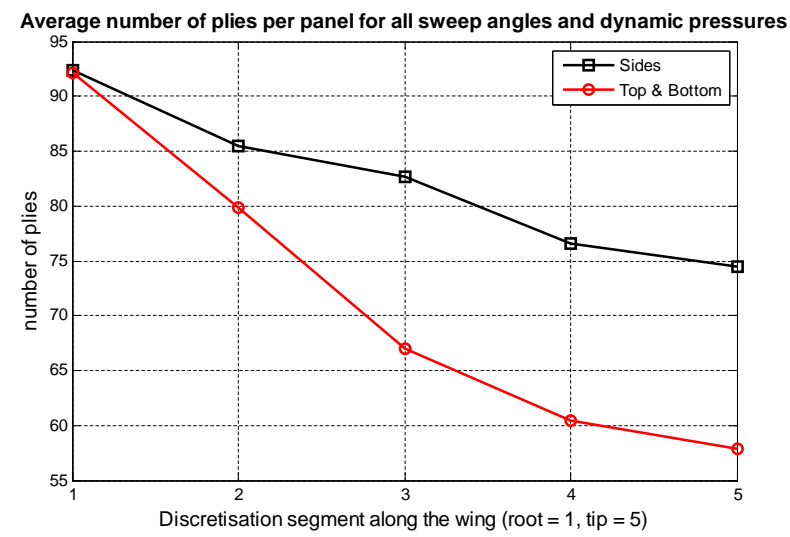

Fig. 7 Average number of plies per panel, for top and side panels. The average is taken over all $\Lambda$ and $M_{\infty}$.

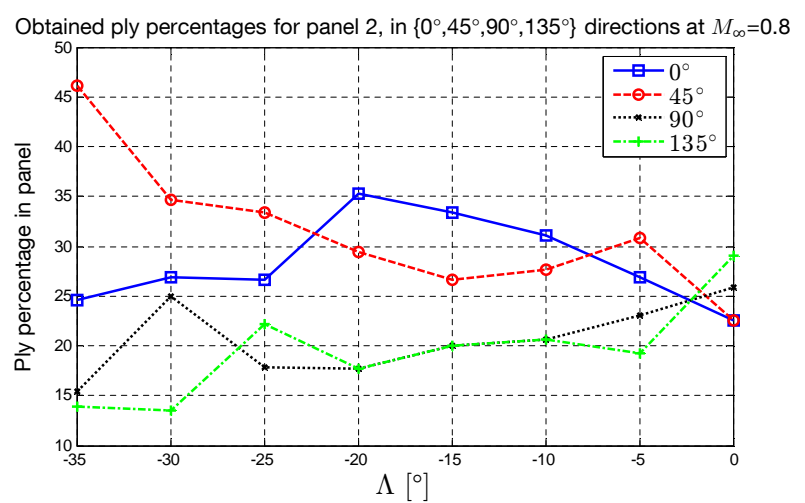

Fig. 8 Ply percentages in top panel of wing discretization segment 2 vs. $\Lambda$, at $M_{\infty}=0.8$. corresponds to an equal number of $-45^{\circ}$ plies in the bottom panel and vice versa. There is a slight trend for more $45^{\circ}$ plies in the top panel as the amount of forward sweep grows larger, especially for the centremost panels $2-4$. This leads to an increase in $\kappa$. Due to the relatively small influence of the tip-most panel, data is especially noisy for this discretization segment and does not show any clear trend towards a certain ply direction.

Furthermore, the panels contain a high percentage of $0^{\circ}$ plies. This was expected, since $0^{\circ}$ plies are most suitable to avoid excessive lift-induced bending. They are oriented parallel to the elastic axis and therefore exhibit their greatest stiffness in that direction.

To provide a measure of the mass reduction achieved by optimization models, the results are compared to a reference wing. This wing consists of quasi-isotropic balanced and symmetric composite ply layups, using the same material and only $\left\{0^{\circ}, \pm 45^{\circ}, 90^{\circ}\right\}$ plies. This removes bending-torsion coupling entirely and avoids comparisons to other materials. Only the panel thicknesses are optimized, using the same general approach and aero-structural models as were used for the optimisation of the anisotropic beam. The reference wing therefore represents an optimized design when no bendtwist coupling is present and the divergence problem is overcome by increased stiffness alone. This makes it possible to estimate weight savings achieved by aeroelastic tailoring. The minimum mass of the uncoupled reference wing was found to be $760.67 \mathrm{~kg}$, for a sweep angle of $-25^{\circ}$ and a cruise Mach number of 0.7 . The stacking sequence optimization obtained a mass of 663.82 $\mathrm{kg}$ for the same configuration, which amounts to a wingbox mass reduction of $13 \%$.

\section{Conclusion}

Modern composite materials allow the design of structurally coupled wings to avoid aeroelastic phenomena such as wing divergence. A preliminary aircraft design method for aeroelastic tailoring of forward-swept composite wings has been presented, which can take into account manufacturing constraints. Bending-torsion coupling was exploited to avoid static divergence by optimizing the stacking sequences of wing panels. Weight savings derived from introducing composite tailoring have been obtained which could be used in the preliminary design stages. The optimization was enabled by using relatively computationally cheap models instead of a high-fidelity FE-CFD aeroelastic analysis. It allows the construction of simple plots and the identification of trade-offs (for example with the angle of forward-sweep) in preliminary design stages. While this gives an indi- 
cation of the weight savings available by applying composite tailoring it should be noted that for real aircraft the thickness of the panels is not necessarily symmetric, with the top panels typically containing more plies to prevent buckling.

Results confirm that positive coupling terms increase the divergence speed. Large percentage fractions (typically $30 \%$ to $35 \%$ ) of $45^{\circ}$ plies in the top panels and $-45^{\circ}$ plies in the bottom panels were found to be responsible for the coupling. Furthermore, the optimized wings also contain many $0^{\circ}$ plies to withstand the lift derived bending. Results were compared to an optimized uncoupled reference wing that only uses quasi-isotropic layups. The configuration made use of an elastic axis sweep of $-25^{\circ}$ at $M_{\infty}=0.7$. The LP-based optimization achieved a wingbox mass reduction of $13 \%$. Results showed an increasing wingbox mass and stiffness for increasing forward-sweep, which was mainly due to the aeroelastic divergence constraint.

The influence of the $V^{D}$ parameters and the final stacking sequence was found to be of negligible importance for the mechanical behavior, compared to the number of plies in each direction. The stacking sequence matters mostly for the assessment of structural failure and therefore allows the stacking sequence to be driven by manufacturability constraints 33. Results indicate that a lower-fidelity structural model using just the panels' $[\boldsymbol{A}]$ matrices could achieve similar results, while allowing to reduce the number of design variables. Additional objectives or constraints could be considered with the additional flexibility that not having to match the $V^{D}$ provides. This includes aileron effectiveness or reversal, flutter speed, cruise range or manufacturing constraints. Accuracy can be improved by using higherorder interpolations of the stiffnesses $E I, G J$ and $K$ between discretization points along the wing.

\section{References}

1. Anderson, J.: Fundamentals of aerodynamics, 5th (in SI units) edn. McGraw-Hill (2011)

2. Armanios, E., Badir, A.: Free vibration analysis of anisotropic thin-walled closed-section beams. AIAA J 33(10) (1995)

3. Barbero, E.J., Lopez-Anido, R., Davalos, J.F.: On the mechanics of thin-walled laminated composite beams. J Compos Mater 27(8), 806-29 (1993)

4. Berci, M., Gaskell, P., Hewson, R., Toropov, V.: Multifidelity metamodel building as a route to aeroplastic optimization of flexible wings. P I Mech Eng C-J Mec pp. 2115-2137 (2011)

5. Berci, M., Gaskell, P., Hewson, R., Toropov, V.: A semianalytical model for the combined aeroelastic behaviour and gust response of a flexible aerofoil. J Fluids Struct 38, 3-21 (2013)
6. Berci, M., Toropov, V., Hewson, R., Gaskell, P.: Multidisciplinary multifidelity optimisation of a flexible wing aerofoil with reference to a small uav. Struct Multidiscip O 50, 683-699 (2014)

7. Bisplinghoff, R., Ashley, H., Halfman, R.: Aeroelasticity. Dover Publications Inc. (1955)

8. Bloomfield, M., Diaconu, C., Weaver, P.: On feasible regions of lamination parameters for lay-up optimization of laminated composites. P Roy Soc Lond A Mat 465, 1123-1143 (2009)

9. Bloomfield, M., Herencia, J., Weaver, P.: Enhanced twolevel optimization of anisotropic laminated composite plates with strength and buckling constraints. Thin Walled Struct 47(11), 1161-1167 (2009)

10. Buhl, H.: Advanced Aerospace Materials. Springer Berlin Heidelberg (2012)

11. Darecki, M., Edelstenne, C., Enders, T., Fernandez, E., Hartman, P., Herteman, J.P., Kerkloh, M., King, I., Ky, P., Mathieu, M., Orsi, G., Schotman, G., Smith, C., Wörner, J.D.: Flightpath 2050 - europe's vision for aviation. Report, European Commission (2011)

12. Diaconu, C., Sato, M., Sekine, H.: Feasible region in general design space of lamination parameters for laminated composites. AIAA J 40(3), 559-565 (2002)

13. Diaconu, C., Sekine, H.: Layup optimization for buckling of laminated composite shells with restricted layer angles. AIAA J 42(10), 2153-2163 (2004)

14. Dillinger, J., Klimmek, T., Abdalla, M., Gürdal, Z.: Stiffness optimization of composite wings with aeroelastic constraints. J Aircraft 50(4), 1159-1168 (2013)

15. Georgiou, G., Vio, G., Cooper, J.: Aeroelastic tailoring and scaling using bacterial foraging optimisation. Struct Multidiscip O 50(1), 81-99 (2014)

16. Gjelsvik, A.: The theory of thin walled bars. Wiley (1981)

17. Hahn, H., Tsai, S.: Introduction to Composite Materials. Taylor \& Francis (1980)

18. Hale, J.: Boeing 787 from the ground up. QTR 0406 (2006). URL boeing. com/commercial/aeromagazine

19. Hellard, G.: Composites in airbus - a long story of innovations and experiences (2008). Available at (10/04/2016): http://www.airbusgroup.com/dam/ assets/airbusgroup/int/en/investor-relations/ documents/2008/presentations/GIF2008/gif2008_ workshop_composites_hellard.pdf

20. Herencia, J., Haftka, R., Weaver, P., Friswell, M.: Layup optimization of composite stiffened panels using linear approximations in lamination space. AIAA J 46(9), 2387-2391 (2008)

21. Herencia, J., Weaver, P., Friswell, M.: Morphing wing design via aeroelastic tailoring. In: 48th AIAA/ASME/ASCE/AHS/ASC Structures, Structural Dynamics, and Materials Conference. Honolulu, Hawaii (2007)

22. Hodges, D., Pierce, G.: Introduction to Structural Dynamics and Aeroelasticity, 2 edn. Cambridge University Press, Cambridge (2011)

23. IJsselmuiden, S., Abdalla, M., Gürdal, Z.: Implementation of strength-based failure criteria in the lamination parameter design space. AIAA J 46(7), 1826-1834 (2008)

24. Jebari, R.: Composite stacking sequence optimisation and aeroelastic tailoring of a forward-swept wing. MSc. thesis, Imperial College London (2015)

25. Johnsen, F.: Sweeping Forward: Developing \& Flight Testing the Grumman X-29A Forward Swept Wing Research Aircraft. NASA Aeronautics Book Series (2013). Available at https://www.nasa.gov/sites/ default/files/files/Sweeping_Forward.pdf 
26. Jones, R.: Mechanics of Composite Materials. Taylor \& Francis (1999)

27. Kobelev, V.: Sensitivity analysis of the linear nonconservative systems with fractional damping. Struct Multidiscip O 33, 179-188 (2007)

28. Lentz, W., Armanios, E.: Optimum coupling in thinwalled closed-section composite beams. J Aerosp Eng 11(3) (1998)

29. Librescu, L., Khdeir, A.: Aeroelastic divergence of sweptforward composite wings including warping restraint effect. AIAA J 26(11), 1373-1377 (1988)

30. Librescu, L., Song, O.: On the static aeroelastic tailoring of composite aircraft swept wings modelled as thin-walled beam structures. Compos Eng 2, 497-512 (1992)

31. Liu, B., Haftka, R., Akgun, M.: Two-level composite wing structural optimization using response surfaces. Struct Multidiscip O 20(2), 87-96 (2000)

32. Liu, B., Haftka, R., Trompette, P.: Maximization of buckling loads of composite panels using flexural lamination parameters. Struct Multidiscip O 26(1), 28-36 (2004)

33. Liu, D., Toropov, V., Barton, D., Querin, O.: Weight and mechanical performance optimization of blended composite wing panels using lamination parameters. Struct Multidiscip O 52(3), 549-562 (2015)

34. Liu, D., Toropov, V., Querin, O., Barton, D.: Bilevel optimization of blended composite wing panels. J Aircraft 48(1), 107-118 (2011)

35. Lobitz, D.: Aeroelastic stability predictions for a mwsized blade. Wind Energy 7(3), 211-224 (2004)

36. Lottati, I.: Flutter and divergence aeroelastic characteristics for composite forward swept cantilevered wing. J Aircraft 22(11), 1001-1007 (1985)

37. Massa, J., Barbero, E.: A strength of materials formulation for thin walled composite beams with torsion. J Compos Mater 32(17), 1560-1594 (1998)

38. MathWorks: Matlab documentation - bvp4c. Available at (10/04/2016): http://uk.mathworks.com/help/matlab/ ref/bvp4c.html

39. Raymer, D.: Aircraft Design: A Conceptual Approach, 4 edn. AIAA Education Series. American Institute of Aeronautics and Astronautics (2006)

40. Salim, H., Davalos, J.: Torsion of open and closed thinwalled laminated composite sections. J Compos Mater 39(6), 497-524 (2005)

41. Setoodeh, S., Abdalla, M., Gürdal, Z.: Approximate feasible regions for lamination parameters. In: 11th AIAA/ISSMO Multidisciplinary Analysis and Optimization Conference. Portsmouth, Virginia (2006)

42. Toray-CFA: T300 data sheet. Available at (02/09/2015): http://www.toraycfa.com/pdfs/T300DataSheet.pdf

43. Tsai, S., Halpin, J., Pagano, N.: Invariant Properties of Composite Materials, pp. 233-252. Technomic, Lancaster (1968)

44. TUM: Die Standardatmosphäre (ISA) (2009). Lehrstuhl f. Luftfahrttechnik. Available at (10/04/2016): https://campus.tum.de/tumonline/ LV_TX.wbDisplayTerminDoc?pTerminDocNr=5322

45. Van Campen, J.: Optimum lay-up design of variable stiffness composite structures. PhD thesis, Technische Universiteit Delft (2011)

46. Weisshaar, T.: Aeroelastic stability and performance characteristics of aircraft with advanced composite sweptforward wing structures. Report, Virginia Polytechnic Institute and State University (1978)

47. Weisshaar, T.: Forward swept wing static aeroelasticity. Report, Virginia Polytechnic Institute and State University (1979)
48. Weisshaar, T.: Divergence of forward swept composite wings. J Aircraft 17(6), 442-448 (1980)

\section{Appendix A Divergence calculation (Ritz method)}

The matrix $[\mathcal{M}]$ (equation 21) is of the size $\left(N_{\theta}+N_{w}\right) \times$ $\left(N_{\theta}+N_{w}\right)$, and is given by:

$$
\begin{aligned}
& {[\mathcal{M}]=-\left[\boldsymbol{A}_{\text {stiff }}\right]^{-1}\left[\boldsymbol{A}_{\text {aero }}\right]} \\
& {\left[\boldsymbol{A}_{\text {stiff }}\right]=\left[\begin{array}{c}
-[\boldsymbol{D}]-[\boldsymbol{E}]] \\
-[\boldsymbol{C}]-[\boldsymbol{D}]
\end{array}\right],\left[\boldsymbol{A}_{\text {aero }}\right]=\left[\begin{array}{l}
[\boldsymbol{K}][\boldsymbol{Q}]] \\
{[\boldsymbol{R}][\boldsymbol{S}]}
\end{array}\right]} \\
& K_{i j}=-\sin (\Lambda) \cos (\Lambda) \widetilde{W}_{i j}^{(2)} \\
& Q_{i j}=\cos ^{3}(\Lambda) \widetilde{W}_{i j}^{(1)} \\
& R_{i j}=-\sin (\Lambda) \cos (\Lambda) \widetilde{W}_{i j}^{(4)}-\sin ^{2}(\Lambda) \cos (\Lambda)\left(\widetilde{W}_{i j}^{(6)}+\widetilde{W}_{i j}^{(8)}\right) \\
& S_{i j}=\cos ^{2}(\Lambda) \widetilde{W}_{i j}^{(3)}+\sin (\Lambda) \cos ^{2}(\Lambda)\left(\widetilde{W}_{i j}^{(5)}+\widetilde{W}_{i j}^{(7)}\right)
\end{aligned}
$$

The matrices $[\boldsymbol{C}],[\boldsymbol{D}],[\boldsymbol{E}],\left[\widetilde{\boldsymbol{W}}^{(k), k=1 \ldots 8}\right]$ are obtained as integrals of the chosen shape functions:

$$
\begin{aligned}
& C_{i j}=\int_{0}^{l} E I \psi_{i}^{\prime \prime} \psi_{j}^{\prime \prime} \mathrm{d} \bar{y} \\
& D_{i j}=\int_{0}^{l} K \psi_{i}^{\prime \prime} \varphi_{j}^{\prime} \mathrm{d} \bar{y} \\
& E_{i j}=\int_{0}^{l} G J \varphi_{i}^{\prime} \varphi_{j}^{\prime} \mathrm{d} \bar{y} \\
& \widetilde{W}_{i j}^{(1)}=\int_{0}^{l} e c c_{l \alpha} \varphi_{i} \varphi_{j} \mathrm{~d} \bar{y} \\
& \widetilde{W}_{i j}^{(2)}=\int_{0}^{l} e c c_{l \alpha} \varphi_{i} \psi_{j}^{\prime} \mathrm{d} \bar{y} \\
& \widetilde{W}_{i j}^{(3)}=\int_{0}^{l} e c_{l \alpha} \psi_{i} \varphi_{j} \mathrm{~d} \bar{y} \\
& \widetilde{W}_{i j}^{(4)}=\int_{0}^{l} c c_{l \alpha} \psi_{i} \psi_{j}^{\prime} \mathrm{d} \bar{y} \\
& \widetilde{W}_{i j}^{(5)}=\int_{0}^{l}\left(e c c_{l \alpha}\right)^{\prime} \psi_{i} \varphi_{j} \mathrm{~d} \bar{y} \\
& \widetilde{W}_{i j}^{(6)}=\int_{0}^{l}\left(e c c_{l \alpha}\right)^{\prime} \psi_{i} \psi_{j}^{\prime} \mathrm{d} \bar{y} \\
& \widetilde{W}_{i j}^{(7)}=\int_{0}^{l} e c c_{l \alpha} \psi_{i} \varphi_{j}^{\prime} \mathrm{d} \bar{y} \\
& \widetilde{W}_{i j}^{(8)}=\int_{0}^{l} e c c_{l \alpha} \psi_{i} \psi_{j}^{\prime \prime} \mathrm{d} \bar{y}
\end{aligned}
$$

The largest positive eigenvalue of $[\mathcal{M}]$ is then used to obtain the divergence dynamic pressure.

\section{Appendix B Lamination parameters}

The material invariant matrices $\left[\boldsymbol{\Gamma}_{i}\right]$ 45] and the material invariants $U_{i}$ [26] are defined as:

$\left[\boldsymbol{\Gamma}_{0}\right]=\left[\begin{array}{ccc}U_{1} & U_{4} & 0 \\ U_{4} & U_{1} & 0 \\ 0 & 0 & U_{5}\end{array}\right],\left[\boldsymbol{\Gamma}_{1}\right]=\left[\begin{array}{ccc}U_{2} & 0 & 0 \\ 0 & -U_{2} & 0 \\ 0 & 0 & 0\end{array}\right]$ 
$\left[\boldsymbol{\Gamma}_{2}\right]=\frac{1}{2}\left[\begin{array}{ccc}0 & 0 & U_{2} \\ 0 & 0 & U_{2} \\ U_{2} & U_{2} & 0\end{array}\right],\left[\boldsymbol{\Gamma}_{3}\right]=\left[\begin{array}{ccc}U_{3} & -U_{3} & 0 \\ -U_{3} & U_{3} & 0 \\ 0 & 0 & -U_{3}\end{array}\right]$

$\left[\boldsymbol{\Gamma}_{4}\right]=\left[\begin{array}{ccc}0 & 0 & U_{3} \\ 0 & 0 & -U_{3} \\ U_{3} & -U_{3} & 0\end{array}\right]$

$U_{1}=\frac{1}{8}\left(3 Q_{11}+3 Q_{22}+2 Q_{12}+4 Q_{66}\right)$

$U_{2}=\frac{1}{2}\left(Q_{11}-Q_{22}\right)$

$U_{3}=\frac{1}{8}\left(Q_{11}+Q_{22}-2 Q_{12}-4 Q_{66}\right)$

$U_{4}=\frac{1}{8}\left(Q_{11}+Q_{22}+6 Q_{12}-4 Q_{66}\right)$

$U_{5}=\frac{1}{8}\left(Q_{11}+Q_{22}-2 Q_{12}+4 Q_{66}\right)$

Since $\theta$ does not vary within a single lamina, the integral expressions (1-3) can be simplified to obtain a summation over all plies within a laminate. By requiring that each ply be of the same thickness $t$, the following expressions can be derived, $N$ being the total number of plies [45]:

$\left[\begin{array}{l}V_{1}^{A} \\ V_{2}^{A} \\ V_{3}^{A} \\ V_{4}^{A}\end{array}\right]=\frac{1}{N} \sum_{k=1}^{N}\left[\begin{array}{l}\cos 2 \theta_{k} \\ \sin 2 \theta_{k} \\ \cos 4 \theta_{k} \\ \sin 4 \theta_{k}\end{array}\right]$

$\left[\begin{array}{l}V_{1}^{B} \\ V_{2}^{B} \\ V_{3}^{B} \\ V_{4}^{B}\end{array}\right]=\frac{2}{N^{2}} \sum_{k=1}^{N}\left(\left(\frac{N}{2}-k+1\right)^{2}-\left(\frac{N}{2}-k\right)^{2}\right)\left[\begin{array}{l}\cos 2 \theta_{k} \\ \sin 2 \theta_{k} \\ \cos 4 \theta_{k} \\ \sin 4 \theta_{k}\end{array}\right]$

$\left[\begin{array}{l}V_{1}^{D} \\ V_{2}^{D} \\ V_{3}^{D} \\ V_{4}^{D}\end{array}\right]=\frac{4}{N^{3}} \sum_{k=1}^{N}\left(\left(\frac{N}{2}-k+1\right)^{3}-\left(\frac{N}{2}-k\right)^{3}\right)\left[\begin{array}{l}\cos 2 \theta_{k} \\ \sin 2 \theta_{k} \\ \cos 4 \theta_{k} \\ \sin 4 \theta_{k}\end{array}\right]$

\section{Appendix C Reduced stiffness matrix}

For each panel, a reduced stiffness matrix is obtained by neglecting the normal stress $N_{s}$ and bending moment $M_{s}$ about $\underline{s}$. The laminates considered here are symmetric. The following relations exist between panel resultant forces and midplane strains and between moments and curvatures [3] 26]:

$\left[\begin{array}{c}N_{z} \\ N_{s} \\ N_{s z}\end{array}\right]=[\boldsymbol{A}]\left[\begin{array}{c}\epsilon_{z} \\ \epsilon_{s} \\ \gamma_{s z}\end{array}\right],\left[\begin{array}{c}M_{z} \\ M_{s} \\ M_{s z}\end{array}\right]=[\boldsymbol{D}]\left[\begin{array}{c}\kappa_{z} \\ \kappa_{s} \\ \kappa_{s z}\end{array}\right]$

Where $[\boldsymbol{A}]$ and $[\boldsymbol{D}]$ are the extensional and bending stiffness matrices of the panel considered, as defined by [26. The two matrices are inverted, their second row and column are neglected, and the $4 \times 4$ resulting matrices are inverted again. The expressions (72) hence become:

$\left[\begin{array}{c}N_{z} \\ N_{s z}\end{array}\right]=\left[\begin{array}{c}A_{z} A_{s z} \\ A_{s z} A_{s}\end{array}\right]\left[\begin{array}{c}\epsilon_{z} \\ \gamma_{s z}\end{array}\right],\left[\begin{array}{c}M_{z} \\ M_{s z}\end{array}\right]=\left[\begin{array}{c}D_{z} D_{s z} \\ D_{s z} D_{s}\end{array}\right]\left[\begin{array}{c}\kappa_{z} \\ \kappa_{s z}\end{array}\right]$

And the reduced matrices are:

$\left[\begin{array}{c}X_{z} X_{s z} \\ X_{s z} X_{s}\end{array}\right]=\left[\begin{array}{cc}X_{11}-\frac{X_{12}^{2}}{X_{22}} & X_{13}-\frac{X_{12} X_{23}}{X_{22}} \\ X_{13}-\frac{X_{12} X_{23}}{X_{22}} & X_{33}-\frac{X_{23}^{2}}{X_{22}}\end{array}\right]$

Where $\mathrm{X}$ can be replaced by $A$ or $D$. 